Reprod. Nutr. Dévelop., 1984, 24 (3), 297-305.

\title{
Technique d'étude de l'activité unitaire des neurones centraux chez le mouton éveillé
}

D. CATALIN

Département de Physiologie et Neurophysiologie (L.A. 205 CNRS), Faculté des Sciences et Techniques St-Jérôme,

rue Henri-Poincaré, 13397 Marseille Cedex 13, France.

Summary. A technique for recording central neuron activity in conscious sheep.

This paper describes a technique for recording central neuron activity in conscious sheep. Using this technique, neuronal activity can be recorded using classical stereotaxic coordinates and without immobilizing the animal's head.

\section{Introduction.}

L'étude de l'activité des neurones centraux chez l'animal éveillé s'est beaucoup développée depuis les travaux princeps d'Evarts (1966, 1968). En effet, l'enregistrement de l'activité unitaire des neurones centraux en condition physiologique, c'est-à-dire chez l'animal éveillé, permet d'approcher plus précisément que sous anesthésie la nature exacte du message nerveux. Dans le cadre des recherches effectuées au laboratoire sur le contrôle nerveux de la déglutition et de la motricité œsophagienne (voir Roman, 1967 ; Car, 1975 ; Jean, 1978; Roman et Gonella, 1981) nous avons mis au point une technique permettant d'enregistrer l'activité des neurones thalamiques du noyau ventropostéro-médian (VPM) chez le mouton éveillé. En effet, les techniques utilisées par plusieurs auteurs chez différentes espèces (Hayward et Vincent, 1970 ; Baldwin et al., 1971 ; Siegel et Lineberry, 1971 ; Vincent et al., 1972 ; Jennings et al., 1978) ne sont pas adaptées à cette étude. La contention rigide de la tête est très mal acceptée chez le mouton éveillé. Par ailleurs, l'utilisation de radiographies et/ou de ventriculographies (Vincent et al., 1972 ; Jennings et al., 1978), outre la nécessité de disposer d'un matériel adapté, ne peut servir que pour l'étude de structures situées à proximité des repères osseux ou des cavités ventriculaires. La cible visée dans notre étude (noyau VPM), de taille réduite, impose une méthode précise de repérage et nous avons choisi d'utiliser la méthode stéréotaxique. 


\section{Principe de la technique (fig. 1).}

$\mathrm{Au}$ cours d'une intervention chirurgicale en conditions aseptiques, sous anesthésie profonde, une ouverture est pratiquée dans le crâne de l'animal, audessus de la zone que l'on se propose d'explorer. Un puits métallique cylindrique est alors scellé sur le crâne, puis est temporairement rendu hermétique grâce à la pose en région supérieure d'un obturateur amovible en téflon.

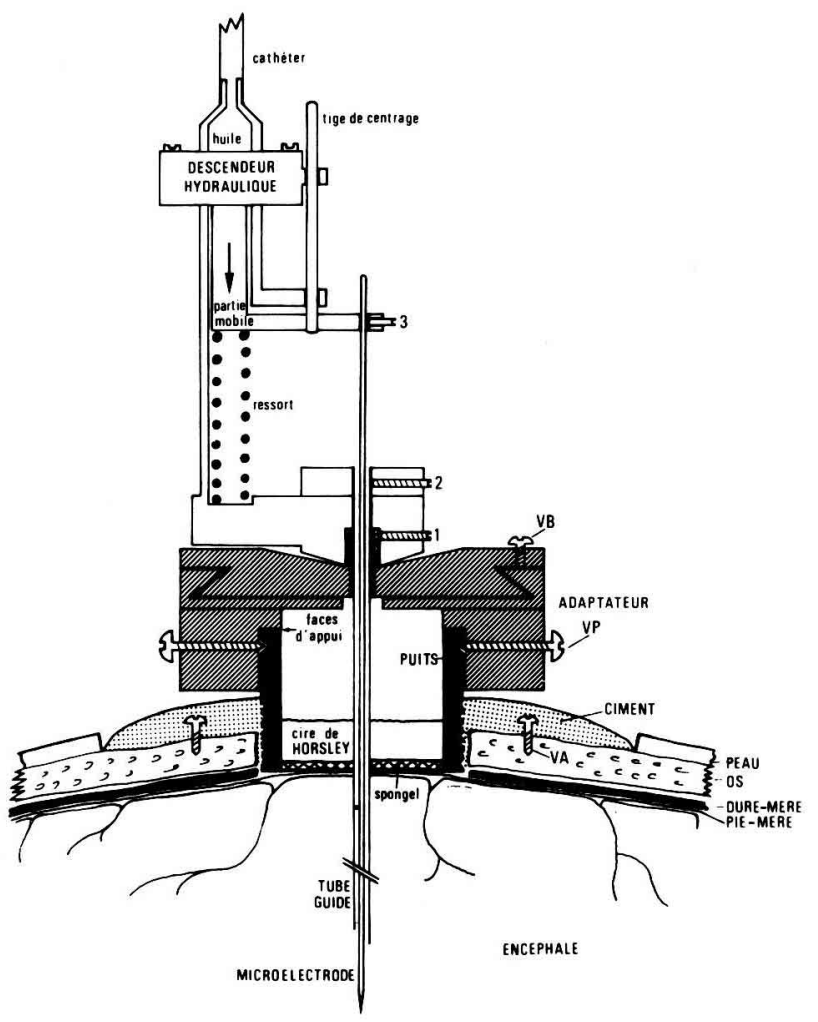

FIG. 1. - Coupe du dispositif d'exploration fixé sur la boîte crânienne.

$1:$ vis de fixation du descendeur hydraulique sur l'adaptateur intermédiaire $; 2:$ vis de solidarisation du tube guide avec le bâti fixe du descendeur hydraulique ; 3 : vis de blocage de la microélectrode sur la partie mobile du descendeur hydraulique.

VA : vis d'ancrage fixée à l'os et englobée dans le ciment de scellement. VB : vis de blocage des 2 chariots de I'adaptateur. VP : vis pointeaux solidarisant l'adaptateur au puits. (Voir les commentaires dans le texte.)

Lors de la phase d'enregistrement sur animal éveillé, l'obturateur est remplacé par un adaptateur destiné à supporter le descendeur de la microélectrode. Cet adaptateur comporte deux chariots mobiles dans deux directions 
orthogonales, ce qui permet le déplacement du descendeur et de la microélectrode dans deux directions. Le descendeur, de type hydraulique, permet le déplacement de l'électrode selon la troisième direction.

\section{Description du matériel.}

1) Puits. - Le puits est constitué par un cylindre en acier inoxydable, comportant une partie externe filetée (fig. 2A). Il est fixé sur le crâne de l'animal au moyen de ciment. Le puits fabriqué doit permettre l'emboîtement de la plateforme intermédiaire, et son blocage dans une position définie. Pour cela, la partie supérieure et externe du puits est creusée d'un sillon circulaire taillé en $V$ recevant les deux vis pointeaux servant à la solidarisation de l'adaptateur intermédiaire. De plus, une vis d'orientation placée dans le corps du puits permet d'orienter le puits et l'adaptateur intermédiaire selon l'axe antéro-postérieur de l'animal. En outre, elle sert à bloquer un obturateur en téflon fermant la lumière du puits lorsque l'animal n'est pas soumis à l'expérimentation.

2) Support du puits. - Pour assurer l'implantation stéréotaxique du puits, un support a été fabriqué (fig. 2A). Ce support comporte une hampe carrée pouvant être fixée sur un descendeur d'électrode à crémaillère utilisé uniquement pendant la préparation chirurgicale de l'animal. Une pièce circulaire fixée à la partie inférieure de la hampe permet d'emboîter et de fixer le puits grâce à la vis $d^{\prime}$ orientation. Le support comporte en outre une tige terminée par une pointe, logée dans un évidement pratiqué dans le corps de la hampe carrée. Cette tige, maintenue par une vis, peut coulisser à l'intérieur de ce logement.

3) Adaptateur intermédiaire. - Ce dispositif d'adaptation placé entre le puits et le descendeur hydraulique est constitué par trois pièces en laiton (fig. 2B) :

- une base fixe $A$ qui vient recouvrir le sommet du puits, une pièce intermédiaire $B$ qui coulisse selon un plan latéral, une pièce supérieure $C$ qui permet la translation antéro-postérieure et la fixation du descendeur hydraulique sur une partie cylindrique centrale. La base $A$ est évidée au même diamètre externe que le puits de façon à assurer un emboîtement maximal de l'assemblage et à réduire ainsi la hauteur supplémentaire introduite par la pièce. Ceci permet de diminuer également la longueur de la microélectrode utilisée ;

- le déplacement de B sur A ou de C sur B est assuré par un assemblage coulissant à queue d'aronde. La coordonnée désirée peut être lue sur des graduations gravées sur chacune des pièces responsables d'une translation. Des vis d'arrêt, situées à la partie supérieure et sur le côté de cet ensemble, permettent le blocage des parties mobiles. Ce type de chariotage double permet un déplacement selon les deux axes antéro-postérieur et latéral, en référence aux repères stéréotaxiques ;

- un logement situé sur un côté de la base fixe permet le passage d'une vis d'orientation assurant le positionnement de l'adaptateur suivant l'axe antéro- 
postérieur de l'animal. De plus, la plateforme $A$ est munie de filetages sur ses faces latérales, ce qui assure sa fixation sur le puits par l'intermédiaire de deux vis pointeaux de solidarisation.

A

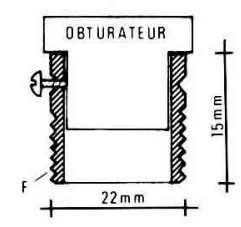

COUPE DU PUITS

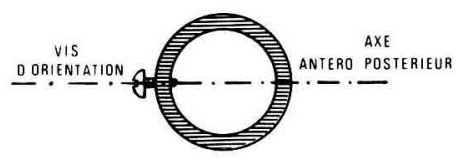

PUITS

VUE DE DESSUS

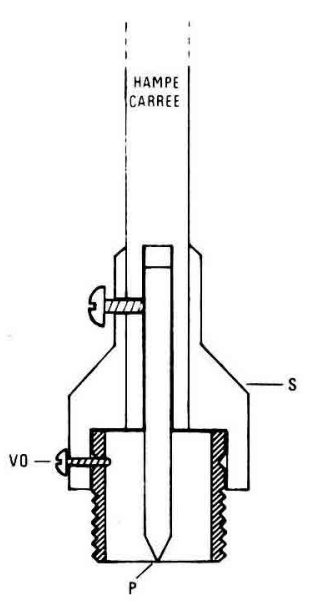

COUPE DU SUPPORT

B

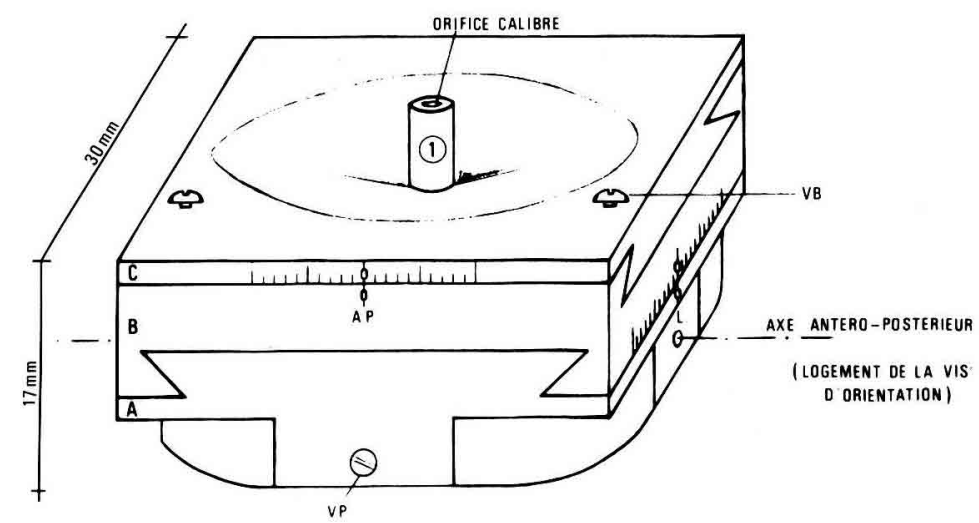

FIG. 2. - Croquis du puits, du support du puits et de l'adaptateur intermédiaire.

En $A$ : $F$ : filetage externe. $S$ : support du puits. V.O. ; vis d'orientation. $P$ : pointe centrale. En $B$ : (1) : partie cylindrique. $A$ : base fixe. $B$ : pièce intermédiaire. $C$ : pièce supérieure. V.B. : vis de blocage de C/B. VP : vis pointeau de solidarisation. (Voir les commentaires dans le texte.)

4) Descendeur hydraulique. - Le microdescendeur hydraulique employé est un appareil du commerce (chronic hydraulic microdrive type 30700, J. H. Wells). 
5) Microélectrodes. - Nous avons utilisé des microélectrodes métalliques en platine-iridium affinées par électrolyse et isolées au moyen de verre (Guld, 1964 ; Catalin, 1982). Des électrodes simplement vernies ne sont pas recommandées. En effet, dans la technique proposée la microélectrode solidaire du descendeur hydraulique coulisse dans un tube guide qui la protège. Lors de la descente des frottements se produisent entre le corps de la microélectrode et le tube guide. Ces frottements sur du vernis produisent un défaut d'isolement après quelques descentes ce qui n'est pas le cas avec un revêtement de verre.

\section{Préparation de l'animal.}

1) Détermination préalable des coordonnées stéréotaxiques du puits. - On détermine tout d'abord le zéro stéréotaxique du centre du puits avant de le positionner, selon les coordonnées choisies, au cours de l'intervention chirurgicale. Le puits est fixé sur son support. La pointe centrale est ajustée au même niveau que la partie inférieure du puits, qui va se trouver au contact de la pie-mère lors de l'implantation. L'ensemble est fixé sur un descendeur d'électrode à crémaillère, solidaire du cadre stéréotaxique, de telle sorte que la vis d'orientation détermine l'axe antéro-postérieur du puits. Le puits est alors enlevé, ce qui rend la pointe centrale apparente. On peut ainsi déterminer le zéro stéréotaxique sur la pointe. Les coordonnées déterminées correspondent au centre du puits. II suffira, lors de I'implantation, de positionner le puits dans le plan de l'atlas choisi (Richard, 1967).

2) Implantation du puits. - Le mouton est profondément anesthésié (fluothane 2 à $3 \%$ ). La tête est fixée dans le cadre stéréotaxique. La peau est incisée de façon à dégager la portion du crâne correspondant à la zone d'implantation. Le descendeur à crémaillère portant le support du puits muni de sa pointe, mais non du puits, est fixé sur le cadre stéréotaxique. II va permettre d'amener la pointe du support du puits au contact du crâne, à l'endroit choisi pour être le centre du puits compte tenu des coordonnées des plans antéro-postérieurs et parasagittaux que l'on se propose d'explorer. Partant de ce repère, l'exérèse de l'os et de la dure-mère est pratiquée sur une portion correspondant aux côtes externes du puits. La dure-mère est incisée puis réséquée en grande partie. La pointe centrale est enlevée du support de puits de façon à ne pas léser la pie-mère au cours de l'implantation. Le puits est alors monté sur son support. La crémaillère du support permet la descente du puits jusqu'à ce que son bord inférieur affleure la pie-mère. Le repère de hauteur, lu sur le vernier du descendeur à crémaillère, indique la hauteur du niveau de la pie-mère. Celle-ci permet alors de connaître la distance entre la surface du cortex et le zéro de l'atlas, et donc de déterminer la profondeur de la structure choisie par rapport à cette surface. Une lame de spongel est intercalée entre le puits et la pie-mère. Le puits est alors scellé à l'os grâce à du ciment dentaire. La vis d'orientation solidarisant le puits à son support est débloquée après la prise du ciment. Le support est retiré laissant le puits sur le crâne. Une petite quantité de cire à trépanation de Horsley, rendue liquide, est coulée par la lumière 
supérieure du puits sur le spongel. Cette cire, une fois durcie, évite la fuite du liquide céphalo-rachidien. Un obturateur en téflon est emboîté dans la lumière du puits et maintenu par la vis d'orientation.

\section{Exploitation de la préparation.}

Elle peut débuter 1 à 2 jours après l'implantation du puits. Le mouton éveillé est installé dans une stalle surélevée. Il est simplement attaché au moyen d'un collier de façon à éviter des mouvements trop importants de la tête. L'obturateur du puits est enlevé, ainsi que sa vis de maintien. L'adaptateur intermédiaire est alors emboîté sur le puits et orienté dans sa position de référence grâce à la vis d'orientation. Après le blocage de la plateforme intermédiaire par les 2 vis pointeaux de solidarisation, la vis d'orientation peut être ôtée. Une microélectrode est fixée sur le microdescendeur hydraulique et protégée par un tube guide solidaire du descendeur. L'ensemble est alors positionné sur l'adaptateur intermédiaire et bloqué sur sa partie cylindrique centrale (voir fig. 1). Le tube guide est sélectionné en fonction de la hauteur du niveau de la pie-mère, déterminée lors de l'intervention chirurgicale, par rapport à la hauteur du zéro stéréotaxique. On peut ainsi définir la profondeur de la structure à explorer par rapport à la surface du cortex. Ceci permet de calculer la longueur du tube guide utilisé afin que son extrémité inférieure soit située environ $3 \mathrm{~mm}$ au-dessus de la cible.

\section{Mise à l'épreuve de la technique proposée.}

Une expérimentation électrophysiologique consistant à étudier l'activité d'un noyau thalamique (le noyau VPM) chez le mouton éveillé a été effectuée sur cinq animaux. Ce noyau constitue le relais thalamique primaire des afférences de la région oro-faciale (Richard et al., 1967). Les résultats préliminaires obtenus indiquent que la cible a bien été atteinte et attestent la validité de la technique. Des réponses unitaires à courte latence ( 4 à $5 \mathrm{~ms}$ ) ont été enregistrées lors de la stimulation des lèvres inférieures ipsilatérales (Fig. 3A). Ces résultats sont tout à fait en accord avec ceux obtenus par Richard et al. (1967) chez l'animal anesthésié. Par ailleurs, les activités de nombreux neurones déchargeant lors de la mastication et/ou lors de la déglutition ont également été enregistrées. Enfin, les contrôles histologiques effectués après électrocoagulation ont confirmé que le noyau VPM avait bien été exploré (fig. 3B).

\section{Conclusion.}

La technique mise au point s'est révélée tout à fait adéquate pour étudier I'activité d'une structure centrale, en l'occurrence celle du noyau VPM du thala- 

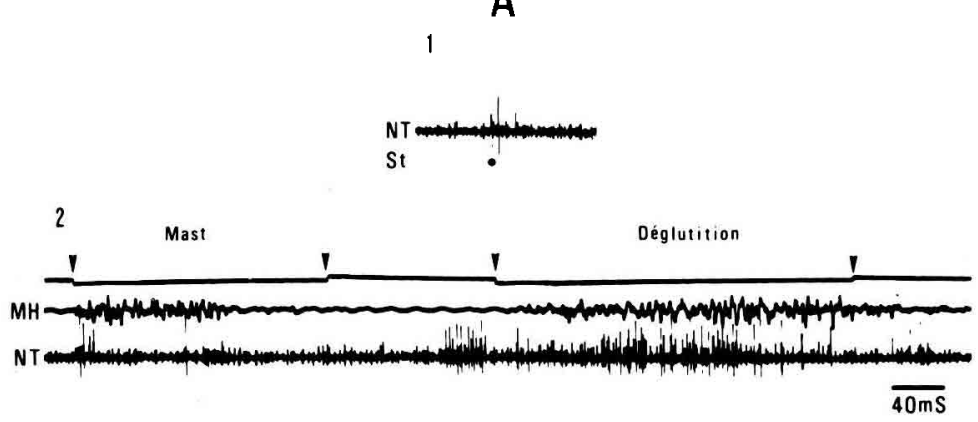

B

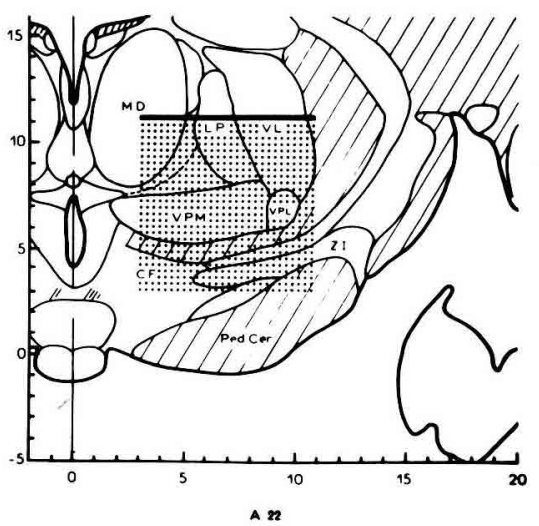

FIG. 3. - Activité d'un neurone thalamique et localisation des zones d'exploration.

En A : MH : EMG du muscle mylohyoïdien. NT : activité du neurone thalamique. st : stimulation. 1 : activation à brève latence $(5 \mathrm{~ms})$ de ce neurone par stimulation (choc unique) de la lèvre inférieure ipsilatérale. 2 : enregistrement de l'activité du même neurone lors de mouvements spontanés de mastications et de déglutitions. Ces mouvements sont indiqués par les déflexions du tracé supérieur (flèches).

En B : La carte représente le plan A22 de I'atlas de Richard (1967). La trame en pointillé délimite la zone explorée avec les microélectrodes. Le trait noir horizontal indique la limite de pénétration des tubes guides. CF : campi foreli. LP : nucleus lateralis posterior. MD : nucleus medialis. Ped. Cer. : pedunculus cerebri. VL: nucleus ventralis lateralis. VPM : nucleus ventralis posterior (P. medialis). VPL : nucleus ventralis posterior ( $P$. lateralis). $\mathrm{ZI}$ : zona incerta.

mus. Elle est bien adaptée au mouton, compte tenu des contraintes imposées à l'animal qui restent minimes (fig. 4). Par ailleurs, les divers dispositifs de fixation utilisés réduisent le temps perdu lors du repositionnement de l'électrode d'une exploration à l'autre. De plus, l'utilisation de la méthode stéréotaxique permet d'effectuer de manière simple une exploration systématique de la structure cible ; le repérage des régions que l'on veut explorer peut se faire sur les plans de l'atlas, 


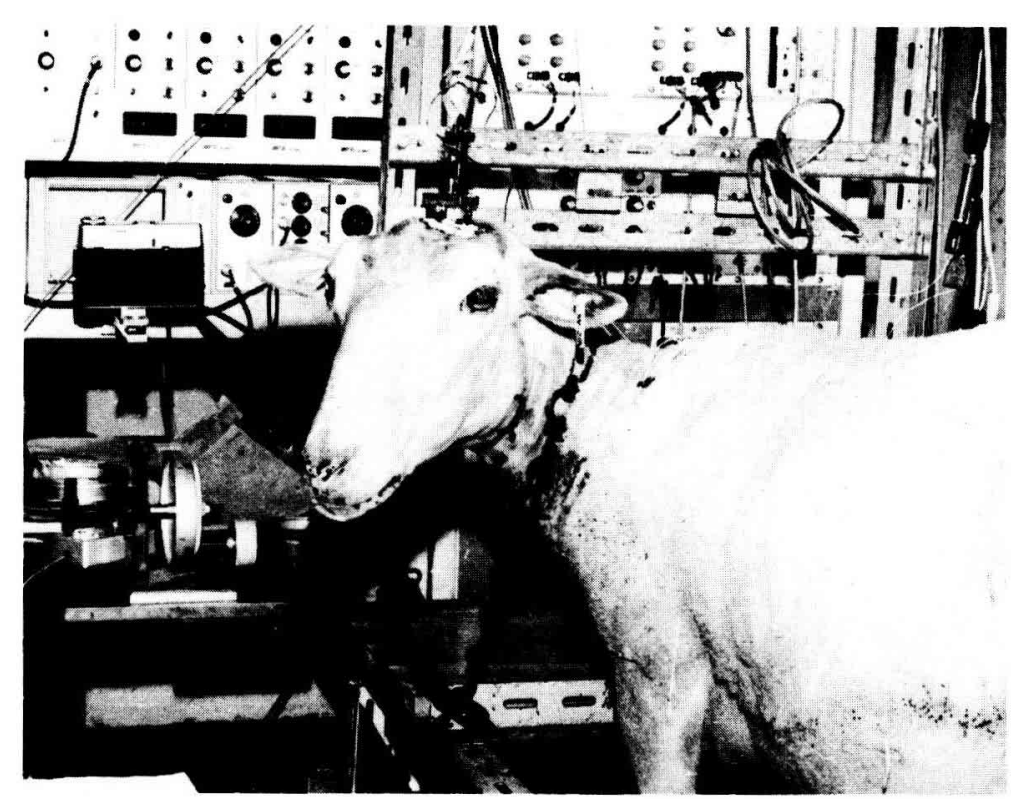

FIG. 4. - Mouton équipé du matériel d'exploration.

comme lors des expérimentations chez les animaux anesthésiés. Cependant, il faut signaler que les structures les plus médianes ne peuvent pas être explorées ; en effet, elles se situent sous le sinus veineux interhémisphérique qui ne peut être récliné que de façon partielle, ce qui limite la zone d'exploration.

Quoi qu'il en soit, cette technique paraît avoir un intérêt d'ordre général puisqu'elle est directement transposable à l'exploration de nombreuses structures du système nerveux central.

Reçu en octobre 1983.

Accepté en décembre 1983.

Remerciements. - A Monsieur le Professeur M. Dussardier pour ses conseils et ses critiques lors de la rédaction de cet article et au Docteur $A$. Jean qui m'a guidé au cours de ce travail.

Cette étude a bénéficié de subventions de I'INRA.

\section{Références}

BALDWIN B. A., SIEGEL J., YATES J. O., 1971. A method for recording unit neural activity in unanaesthetized sheeps and goats. Physiol. Behav., 7, 935-938.

CAR A., 1975. La commande corticale de la déglutition. Th. Doct. ès-sci., Marseille, $149 \mathrm{pp}$.

CATALIN D., 1982. Mise au point de techniques pour l'enregistrement de l'activité unitaire des neurones thalamiques chez le mouton éveillé. Diplôme Etudes sup., Marseille, 36 pp. 
EVARTS E. V., 1966. Methods for recording activity of individual neurons in moving animals, 241-250. In R. F. RUSHMER, Methods in medical research. Year Book med. Publ., Chicago.

EVARTS E. V., 1968. A technique for recording activity of subcortical neurons in moving animals. EEG clin. Neurophysiol., 24, 83-86.

GULD C., 1964. A glass-covered platinum microelectrode. Med. electron. Biol. Engng., 2, 317-327. HAYWARD J. N., VINCENT J. D., 1970. Osmosensitive single neurons in the hypothalamus of unanesthetized monkeys. J. Physiol. (London), 210, 947-972.

JEAN A., 1978. Contrôle bulbaire de la déglutition et de la motricité cesophagienne. Th. Doct. ès-sci., Marseille, $278 \mathrm{pp}$.

JENNINGS D. P., HASKINS J. T., ROGERS J. M., 1978. Comparison of firing patterns and sensory responsiveness between supraoptic and other hypothalamic neurons in the unanesthetized sheeps. Exp. Brain Res., 149, 347-364.

RICHARD Ph., 1967. Atlas stéréotaxique du cerveau de brebis (préalpes du sud). INRA Edit., Paris.

RICHARD Ph., AUFFRAY P., ALBE-FESSARD D., 1967. Activités thalamiques évoquées par des stimulations somatiques chez le mouton anesthésié au chloralose. EEG clin. Neurophysiol., 23, 401-409.

ROMAN C., 1967. La commande de la motricité cesophagienne et sa régulation. Th. Doct. ès-sci., Marseille, $181 \mathrm{pp.}$

ROMAN C., GONELLA J., 1981. Extrinsic control of digestive tract motility, 289-332. In L. R. JOHNSON, Physiology of the gastrointestinal tract, Raven Press, New York.

SIEGEL J., LINEBERRY C. G., 1971. A method for recording single unit activity from unanesthetized chronic cats. Physiol. Behav., 6, 607-608.

VINCENT J. D., ARNAULD E., BIOULAC B., 1972. Activity of osmosensitive single cells in the hypothalamus of the behaving monkey during drinking. Exp. Brain Res., 44, 371-384. 